\title{
ECOULEMENT DE L'EAU AU-DESSOUS D'UNE VANNE ET FORME RATIONNELLE DE LA SURFACE D'APPUI DE LA VANNE
}

\section{ETUDE THÉORIQUE ET EXPÉRIMENTALE}

\author{
par le Professeur Jean SMETANA
}

Ingénieur-Docteur de l'Université de Prague

\section{I. - LE PROBLÈME}

\section{Les différents types d'écoulement par l'ouver-} ture au-dessous d'une vanne.

\section{A - par une ouverture:}

a) soit émergeante au-dessus de l'eau à l'aval;

b) soit noyée partiellement ou complètement.

$B$ - avec contraction du courant :

a) soit sur toute la périphérie de l'ouverture:

b) soit sur une partie de cette périphérie.

C - par un courant :

a) soit libre, c'est-à-dire limité de toutes parts par l'air:

b) soit guidé par un canal.

D - par un courant torrentiel :

a) soit à surface libre ;

b) soit à jet noyé.

\section{2.' Choix du cas.}

Afin de pouvoir considérer de la manière la plus parlante l'écoulement théorique et expérimental de l'eau au-dessous d'une vanne pour cet écoulement, nous allons choisir le cas qui est représenté par les figures 1 et 2 . La vanne est placée dans un canal qui a des parois verticales et un fond horizontal. La largeur de la vanne et la largeur du canal à l'amont et à l'aval de la vanne sont égales. La vanne a une surface d'appui ayant la forme d'une arête horizontale bisøautée.

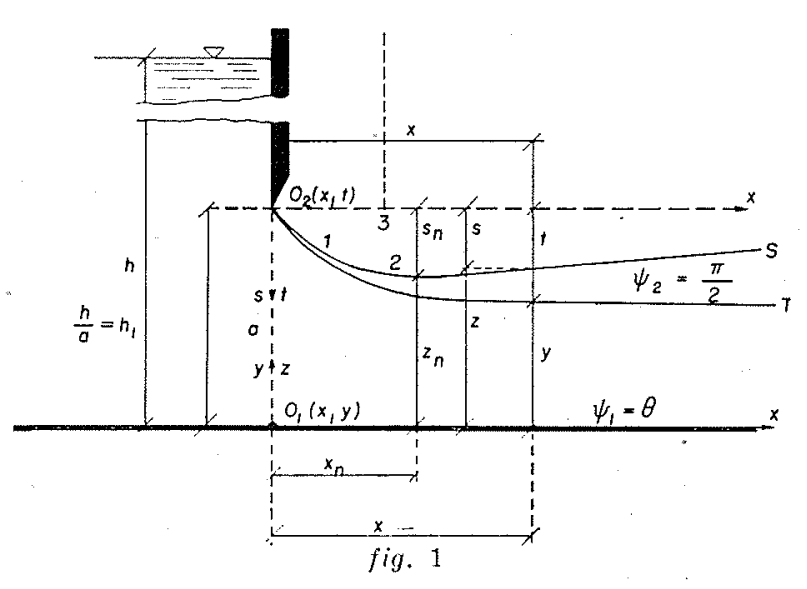

Ecoulement torrentis à surface libre

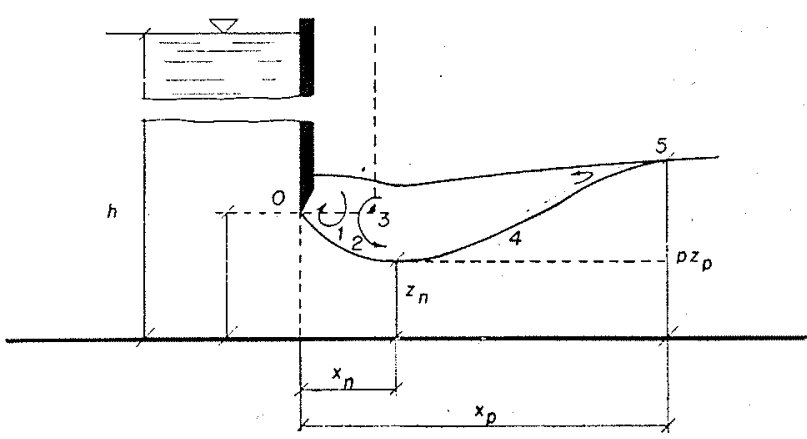

fig. 2

Ecoulement torrentiel à ressaut noyé

L'écoulement considéré a lieu alors par une ouverture noyée: $(\mathrm{Ab})$ avec contraction provoquée par l'arête supérieure de l'ouverture rectangulaire d'écoulement (Bb), par un courant guidé $(\mathrm{Cb})$. On a représenté sur la figure 1 un courant torrentiel à surface libre (Da) avec la surface libre de l'eau suivant la courbe $S$; sur la figure 2 le courant est recouvert (Db) par un ressaut noyé. 


\section{3" Installations d'essai.}

Les figures 3 et 4 montrent le canal du laboratoire hydrotechnique à Prague Podbaba, dans lequel ont été effectués les essais en question. $L_{a}$ figure 5 donne une photographie du même canal avec la vanne remontée de 0,08 mètres au-dessus du fond du canal.

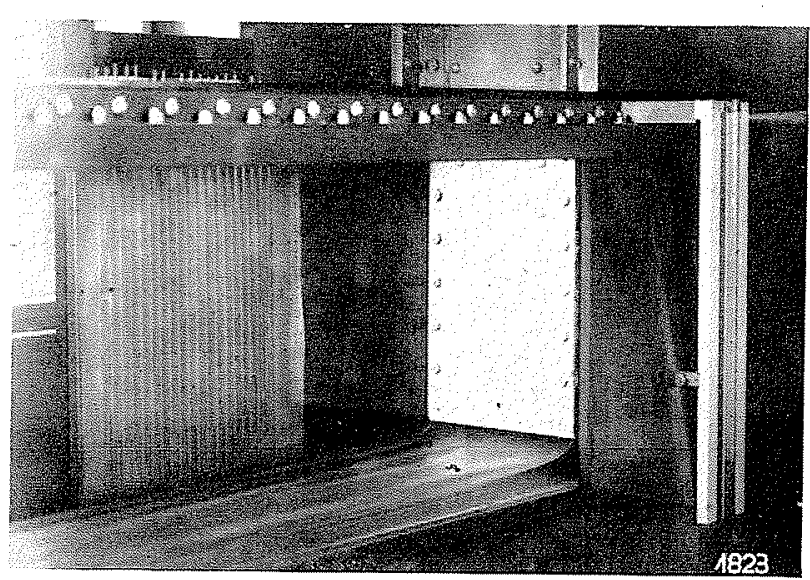

fig. 5

Ecoulement torrentiel à surface libre

La partie du canal d'essai à l'amont de la vanne a une hauteur de $1 \mathrm{~m} .70$ et, sur une longueur de $1 \mathrm{~m} .24$ à l'amont de la vanne, une longueur de $0 \mathrm{~m} .35$; le canai est exécuté en béton et en tôle. La partie du canal à l'aval de la vanne a également une largeur de $0 \mathrm{~m}$. 35. Sa longueur est de 6 mètres et sa hauteur de $0 \mathrm{~m}$. 70. Cette partie du canal a des parois verticales en verre ; son fond horizontal en tôle est recouvert d'une couche lisse de peinture. La vanne même est un tablier vertical en fer terminé en bas par une arête horizontale biseautée. On lit la hauteur d'élévation de la vanne sur une échelle millimétrique avec un vernier. Le débit de l'eau à l'amont de la vanne est mesuré d'une manière rigoureuse par un déversoir triangulaire de Thomson. La hauteur du niveau à l'amont de la vanne est mesurée dans des tubes en verre sur des échelles graduées en millimètres avec estimation de la fraction du millimètre; ces tubes communiquent avec l'eau à l'amont de la vanne par des orifices du fond du canal. Toutes les hauteurs du niveau à l'aval étaient mesurées au moyen d'une pointe mobile avec vernier au dixième de millimètre.

\section{Principe du problème.}

Nous expliquerons le mieux l'objet du problème par l'essai suivant:

Soulevons la vanne de la hauteur a au-dessus du fond. II en résulte une ouverture rectangulaire de hauteur a et de largeur b (fig. 3 et 4 ). Etablissons dans le canal un débit constant tel que le niveau de l'eau à l'amont de la vanne se

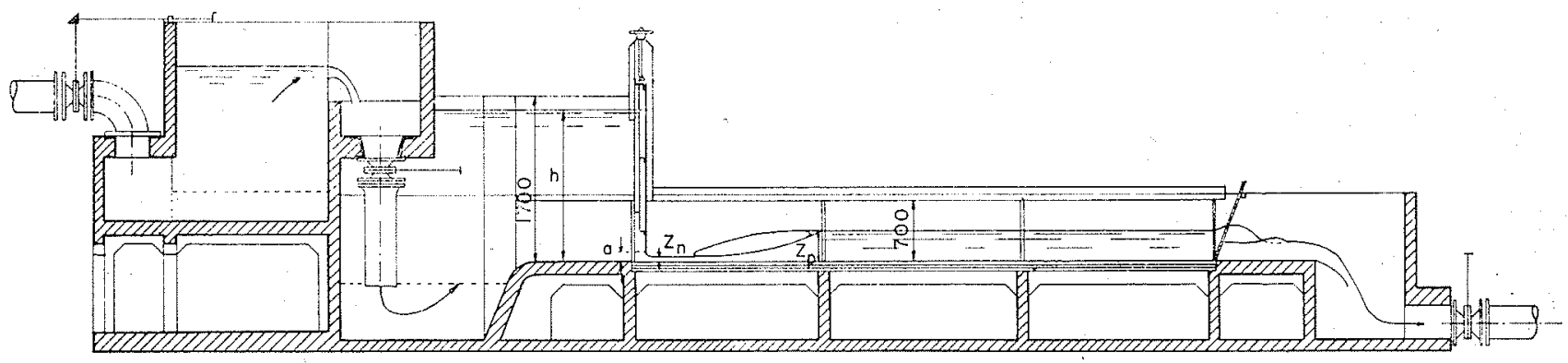

fig. 3

Coupe ingitudinale du canal d'essai

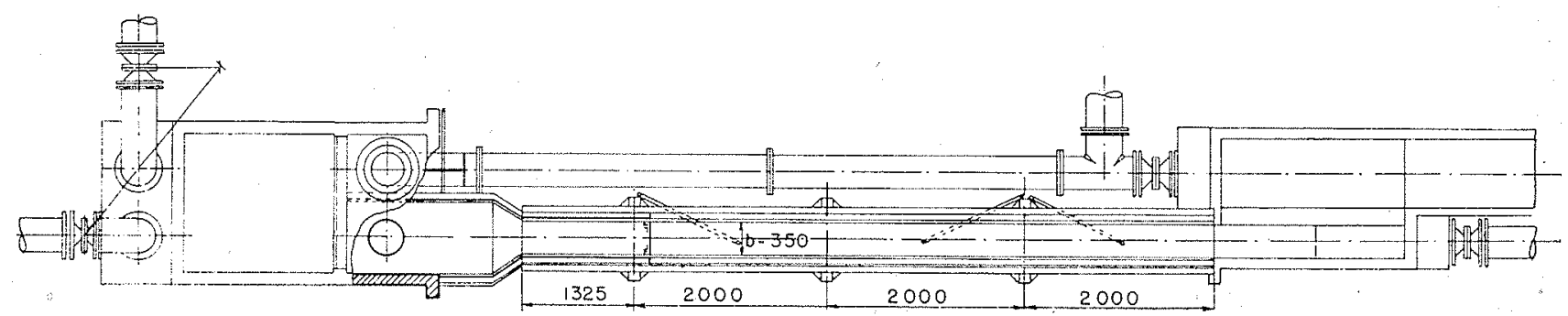

fig. 4

Vue en plan du canal d'essai 
maintienne à la hauteur $h$. Le niveau du courant torrentiel à l'aval de la vanne (fig. 1) s'abaisse suivant la courbe $S$, jusqu'à ce qu'il atteigne à la distance $x_{n}$ la hauteur minimum $z_{n}$ après quoi il remonte très lentement. II remonte parce que la vitesse du courant diminue à cause de la rugosité du fond et des parois.

Afin de créer un courant d'eau recouvert d'un ressaut noyé (fig. 2), il faut réhausser le niveau aval à la hauteur $p z_{p}$ qui est $p$ fois plus grande que la hauteur $z_{p}$ correspondant au ressaut dénoyé (fig. 3). Nous appellerons le nombre $p>1$ le degré de noyage du ressaut. Comme d'après mes travaux antérieurs $(1,2)$ *) le niveau du ressaut remonte suivant la courbe 45 (fig. 2) on a:

$$
x_{p}-x_{m}=\left(p z_{p}-z_{n}\right)
$$

tant que le degré de noyage $p$ n'est pas trop grand.

Si nous remplaçons l'arête biseautée de la vanne par une large surface d'appui horizontale 0.3 (fig. 1), il se produira entre cette surface et le niveau du courant un espace en forme de coin d'où l'air sera entrainé par le frottement hors de cet espace, de manière que la pression $p_{1}$ qui y règne et qui est rapportée au vide absolu soit inférieure à la fression $p_{0}$ de l'atmosphère environnante. Nous appellerons la valeur négative de la différence $p_{1}-p_{01}$ la dépression ou aspiration. Si $F$ est la grandeur de la surface d'appui horizontale, la force verticale $V$ due à la dépression et sollicitant la vanne vers le bas est :

$$
V=\left(p_{1}-p_{0}\right) F
$$

La dépression $p_{1}-p_{0}$ est d'autant plus grande que la vitesse du courant est plus grande, et la chute de la vitesse de l'air entrainé par la surface de l'eau diminue d'autant plus rapidement que l'espace 0,123 a une hauteur plus faible. Afin de compenser la dépression qui s'est créée, l'air extérieur pénètre dans le milieu raréfié et cela d'une manière pulsatoire. De ce fait, la force $V$ varie, produit une charge variable et dans certains cas, fait vibrer le corps de la vanne.

Si le courant sortant de dessous la vanne est noyé (fig. 2), on constatera dans l'espace $O_{2}, 123$ les indices de la cavitation du courant. L'arrivée de l'air extérieur est supprimée aux grandes valeurs du degré de noyage $p$ et aux faibles valeurs de $P$ considérablement entravée. Aux faibles valeurs de $P$, au-dessus de l'endroit de profondeur minimum $z$ le remous sur le courant

\footnotetext{
:) Les chiftres entre parenthèses indiquent les références b.bliographiques citées à la fin de cet ouvrage.
}

sortant de dessous la vanne est crevé par des vortex qui introduisent de manière pulsatoire un peu d'air extérieur (5).

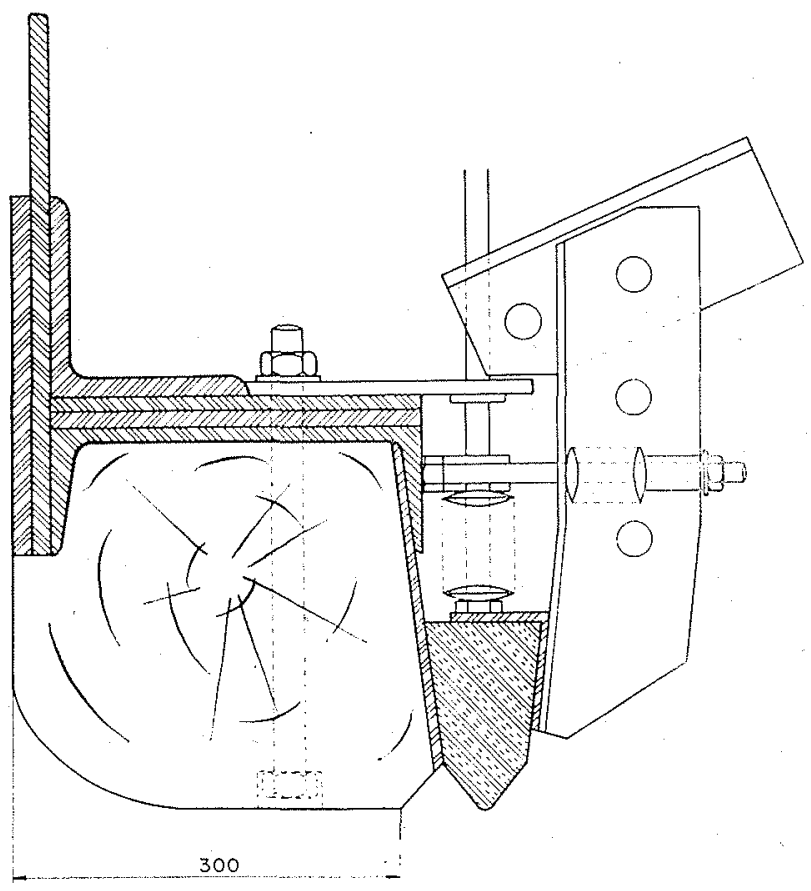

fig. 6

Poutre d'appui de la vanne du barrage sur la Weser, à Dörverden

La force $V$ atteint de grandes valeurs. Ainsi, par exemple, sur la surface d'appui horizontale de la vanne du larron de l'écluse à Stechovice, que nous citerons comme un des résultats de l'application pratique de ces recherches, cette force aurait été de 5 tonnes pour la surface de $2 \mathrm{~m}$. de longueur et de $0 \mathrm{~m}$. 30 de largeur et pour une dépression $p_{1}-p_{0}=-0,8 \mathrm{~kg} . / \mathrm{cm}^{2}$. Les moteurs auraient à l'ouverture de la vanne à supporter une plus grande charge et de plus, la force aurait varié de manière pulsatoire. En outre, lorsqu'il y a de la cavitation, les pièces métalliques sont fortement détériorées.

Enoncé $1: *$ ) - II serait très avantageux pour la construction des vannes et surtout pour leurs dispositifs de levage, que la force variable de dépression ne se produise pas du tout audessous des vannes.

\section{$5^{\circ}$ Technologie de la surface d'appui d'une vanne STONEY.}

L'arête biseautée d'une vanne suffisamment éloignée de la charpente aurait empêché la for$* *$ Les constatations et les conclusions sont résumées par
des iénoncés" dont l'ensemble donne un résumé de ces reche:ches. 
mation de la dépression surtout dans le cas d'un courant à surface libre. Mais nous devons donner à la surface d'appui une largeur définie afin qu'elle puisse transmettre le poids propre de la vanne par un nombre convenable de $\mathrm{kg} / \mathrm{cm}^{2}$ à la crête du barrage.

Afin que la vanne se pose élastiquement et pour qu'elle soit étanche, on utilise le plus souvent une poutre d'appui en chêne qui s'use peu et dure longtemps.

Le bois de chêne soumis à une pression normale aux fibres a une limite de résistance de 127 à $178 \mathrm{~kg} . / \mathrm{cm}^{2}$.

Mais le bois de chêne, s'il est tantôt dans l'eau, et tantôt au-dessus, ou bien d'un côté dans l'eau et de l'autre à l'air, d'une part se tord et d'autre part se fissure. Afin que ces deux inconvénients pèsent moins, on choisit pour la poutre d'appui, surtout si la longueur est grande, une grande section. Afin que la poutre se déforme le moins possible, elle est munie en haut d'un fort fer profilé, renforcé éventuellement encore par un plat et une équerre (fig. 6).

La poutre d'appui de grandes vannes STONEY a une largeur horizontale de $0 \mathrm{~m} .25,0 \mathrm{~m} .30$ et plus.

Si la poutre d'appui se tord, elle n'assure plus bien l'étanchéité. II est particulièrement important qu'elle donne une parfaite étanchéité, lorsque la vanne s'appuie sur la crête d'un barrage émergeant. Si elle n'assure pas l'étanchéité, il y a non seulement une perte d'eau désagréable, mais surtout le gel qui se produit en hiver. Pour cette raison, on a employé, par exemple, dans le barrage sur la Weser à Dörwerden (fig. 6) pour l'étanchéité une bande de caoutchouc qui peut être appliquée par des vis aux endroits où se manifeste le manque d'étanchéité. Ce procédé c'assurer l'étanchéité a été couvert par un brevet (11).

\section{6" Solution actuelle du problème de la forme de la surface d'appui.}

La poutre d'appui est d'habitude prismatique et a à l'amont la forme d'un quart de cylindre circulaire ou elliptique. Le rapport de l'axe vertical à l'axe horizontal de l'ellipse est $3: 5$. La surface d'appui horizontale est un plan tangent à la surface cylindrique (fig. 6). Sa largeur est d'habitude dans les grandes vannes de $10-12 \mathrm{~cm}$., de manière que lorsque la vanne est fermée, la pression ne s'exprime que par quelques $\mathrm{kg} . / \mathrm{cm}^{2}$.
Cette forme, comme on le verra clairement à la fin, n'empêche pas la dépression et ses conséquences, et dans certaines positions d'ouverture de la vanne, ne les diminue pas; cependant elle est parfais recommandée (11).

Parmi d'autres suggestions concernant l'éxécution avantageuse de la surface d'appui d'une vanne, on peut encore signaler les études du $D^{r}$ Ch. KEUTNER $(12,13)$. Le $D^{r}$ KEUTNER a constaté dans ses études:

$1^{\circ}$ - La courbe de contraction de l'écoulement au-dessous de l'arête biseauté d'une vanne es: différente pour chaque ouverture de la vanne; elle dépend de la grandeur du levage de la vanne. L'équation empirique de ces courbes lui paraît différente pour différentes valeurs a de l'ouverture de la vanne. Son équation pour les ordonnées du point le plus bas des courbes en question est avec nos notations suivant la figure I:

$$
s_{n}=0,47 \text { a } 1,08
$$

$2^{\circ}$ - Afin que la surface inférieure de la vanne ne soit pas soumise à une dépression, il faut lui donner la forme d'un volet ayant comme profil le quart d'une ellipse qui, à la montée de la vanne, taurnerait autour d'un axe horizontal.

Le D KEUTNER ne donne pas de détails sur la construction de ce dispositif qui n'est pas simple, de sorte qu'il est difficile de se représenter une réalisation réussie. J'ignore si le volet de KEUTNER a été employé dans la pratique.

\section{$7^{\circ}$ Principe de l'idée de l'auteur.}

La courbe de contraction de l'écoulement à la sortie de dessous de la vanne dépend, comme nous le verrons plus loin, non seulement de la hauteur a de l'auverture, mais aussi du rapport $h_{1}=h$ : a de la hauteur $h$ de l'eau en amont de la vanne à la hauteur a de l'ouverture. Désignons le rapport $h_{1}$ par « charge relative».

Si nous levons la vanne depuis a $=0$ à la valeur maximum $a_{3}$ et si la hauteur $h$ de l'eau en amont de la vanne reste constante comme on le trouve souvent dans les ouvrages hydrauliques, la «charge relative» $h_{1}$ diminue continuellement et de ce fait les courbes de contraction varient.

Supposons que nous ayons toutes les courbes de contraction tracées sur la figure 7. Si nous donnons à la surface d'appui de la vanne la forme de la courbe 3 la plus basse, il s'exercera sur la surface d'appui de la vanne dans la posi- 


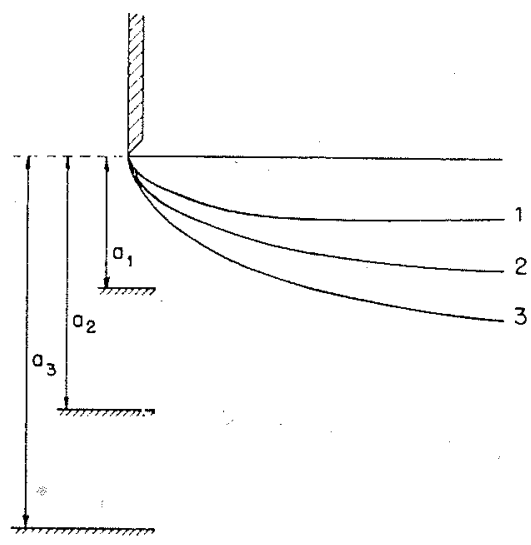

fig. 7

Faisceau des courbes c'écoulement aux différentes ouvertures de la vanne

tion correspondant à la courbe 3 la pression $\mathrm{p}_{1}=\mathrm{p}_{0}$, c'est-à-dire $\mathrm{p}_{1}-\mathrm{p}_{0}=\mathrm{O}$; alors, il n'y aura pas de cavitation ni d'aspiration du courant sortant de dessous la vanne. Dans toute position plus basse de la vanne, on $a: p_{1}-p_{0}>0$, c'està-dire qu'il se produira une poussée verticale.

Enoncé 2 : Il serait avantageux de donner à la surface d'appui de la vanne une forme telle que, dans toute position de la vanne, il y ait sur elle une poussée verţicale. La poussée élimine la cavitation et diminue l'effort nécessaire au levage de la vanne. Et lorsque, pendant le levage de la vanne, elle variera, cette variation se produira d'une manière continue, progressive, et sans faire vibrer la vanne, ce qui est particulièrement indésirable.

Dans quelle mesure ef comment il est possible de réaliser cette idée et quels sont les facteurs dont il faut dans ce but tenir compre. Voici l'objet de ces recherches théoriques et expérimentales.

\section{II. - THÉORIE}

\section{8? Les conditions du mouvement à potentiel de vitesse.}

Tout d'abord, il faut connaître la forme des courbes de contraction ou d'écoulement, comme nous les appellerons par la suite. Nous pouvons l'établir théoriquement, s'il s'agit d'un mouvement plan à potentiel de vitesse. Comme dans la suite, nous comparerons la forme théorique de la courbe d'écoulement avec la forme réelle relevée expérimentalement, rappelons les hypothèses dans lesquelles le mouvement d'un liquide a un potentiel de vitesse et la solution théorique connue pour celui-ci.

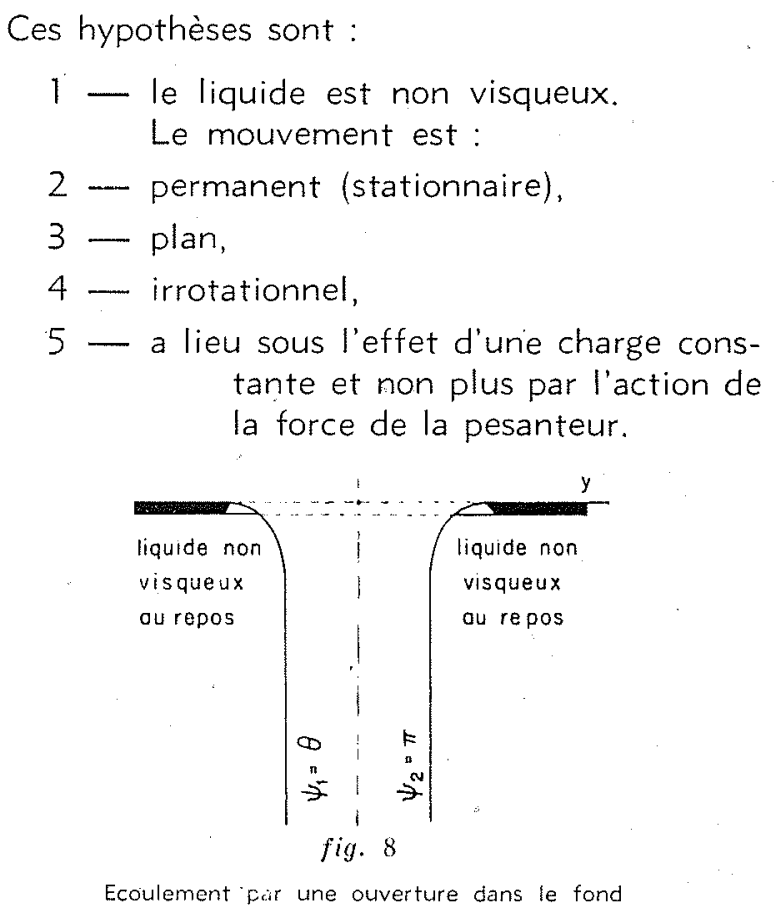

Afin que ces conditions soient réalisées, la théorie (9) suppose qu'il s'agit de l'écoulement cun liquide non visqueux par une fente étroite et allongée dans le fond horizontal d'un réservoir infiniment grand qui pénètre dans un autre liquide non visqueux de même densité (fig. 8).

\section{9" Comment sont réalisées les conditions du mouvement à potentiel de vitesse dans l'installation d'essai utilisée.}

1 - La non viscosité du liquide est exigée afin que la vitesse soit constante dans toute la section du courant, donc au fond et contre les parois. Si la vitesse des particules d'eau est partout la même, la viscosité n'entre même pas en cause. Etant donné que les parois et le fond du canal sont très lisses, le gradient de la vitesse est très grand. La figure 9 montre la variation de la vitesse de l'eau dans le canal d'essai, depuis la surface jusqu'au fond, et d'une paroi à l'autre, qui a été mesurée à l'aide d'un tube de Pitot pour l'ouverture de la vanne $a==0 \mathrm{~m}$. $15 \mathrm{et}$ pour la hauteur de l'eau en amont de la vanne $h=0 \mathrm{~m}$. 42. Les figures sont presque des rectangles. Donc la condition 1 est pratiquement réalisée pour la majeure partie de la hauteur et de la largeur du courant sortant de dessous la vanne.

2 - Le dispositif de l'installation d'essai permet d'avoir un écoulement permanent.

3 - Comme le canal en amont et en aval de 
la vanne et l'ouverture ont la même largeur, il est possible d'avoir un écoulement plan et cela dans des plans verticaux et parallèles à l'axe longitudinal du canal.

4 - Le caractère irrotationnel du mouve. ment dépend des trois premières conditions, et de ce que les particules d'eau passent du repos au mouvement progressivement, sans heurt. II est difficile de s'assurer dans ce cas jusqu'à quelle mesure l'hypothèse du mouvement irrotationnel est remplie. J'ai montré en (6) comment la turbulence ou la non turbulence sont établies expérimentalement.

$0.150 \mathrm{~mm}$

$n=420 \mathrm{~mm}$

$z_{n}=921 \mathrm{~mm}$

$v=2.566 \mathrm{~m} / \mathrm{sec}$
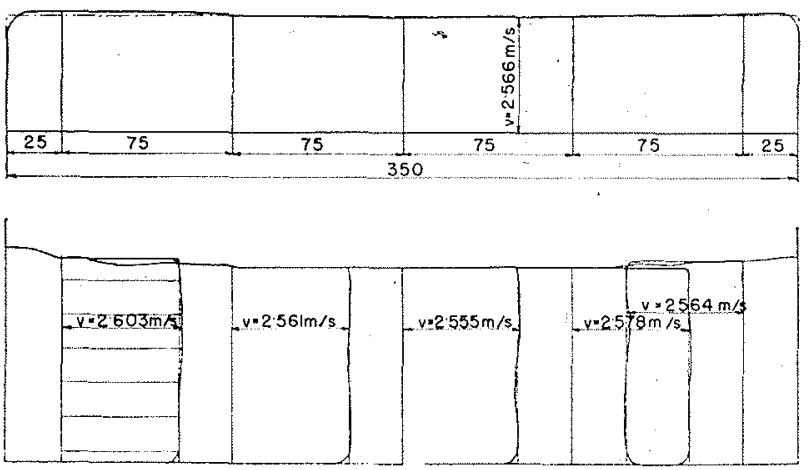

fig. 9

Répartition de la vitesse de l'eau dans le canal d'essai

5 - L'écoulement dans le canal horizontal se fait sous la charge constante $h-z_{n}$ (fig. 1), car il s'agit même dans le cas de courant à surface libre, d'une ouverture immergée dans l'eau d'aval, la profondeur de. l'eau d'aval étant $z$. Du fait, que l'écoulement se fait dans un canal a fond horizontal, la force de la pesanteur est entièrement annulée par la réaction du fond et n'a aucun effet sur le mouvement ultérieur.

La plus grande différence entre les hypothèses théoriques et la réalité de notre dispositif d'essai est toutefois dûe à trois circonstances:

a - le réservoir, c'est-à-dire la partie du canal en amont de la vanne, est relativement petit, surtout aux petites valeurs de la charge relative $h_{1}$, il $y$ a ici un effet sensible de la vitesse d'arrivée dans le réservoir vers la vanne.

b - la rugosité du fond et des parois a comme résultat que la hauteur minimum $z_{n}$ du jet sortant de dessous la vanne est à une distance finie à l'aval de la vanne, alors que théoriquement, elle se trouve à l'infini.

c - bien que les essais étaient effectués de manière que le courant d'eau soit le plus stable et le plus tranquille possible, il n'était pas possible - surtout pour certaines charges relatives $h_{1}$ - d'éviter certains phénomènes pulsatoires qui rendent difficiles les mesures.

Pour les raisons indiquées, la vérification des essais par la théorie et bien entendu, de la théorie par les essais, est très recommandée.

Enoncé 3 : La courbe théorique d'écoulement, lorsqu'elle traduit correctement la loi du mouvement d'un liquide non visqueux dans une conduite idéale, représente l'enveloppe des courbes réelles d'écoulement pour la viscosité du liquide et la rugosité du canal tendant vers zéro, et pour la charge relative croîssant à l'infini.

\section{0" L'équation de la courbe théorique $\mathbf{T}$ de l'écoulement.}

Afin de ne pas être obligé de donner tout un exposé de l'écoulement avec potentiel en se basant sur la théorie des fonctions analytiques, partons des équations différentielles et des équations de son intégration illimitée, que donne l'hydrodynamique théorique (9) pour l'écoulement de l'eau par une ouverture dans le fond.

Considérons le mouvement dans le plan déterminé par les axes de coordonnées $x$ et $y$ conformément à la figure 8 . Si on désigne par:

$$
\begin{aligned}
& \text { le potentiel de la vitesse, } \\
& \text { \la fonction du courant, } \\
& \text { e. la base des logarithmes népériens, }
\end{aligned}
$$
la ligne de courant extrême du jet, que nous appellerons la courbe d'écoulement théorique $T$ est donnée dans les conditions antérieurement exposées par les équations différentielles:

$$
\begin{aligned}
& \frac{d x}{d \phi} \quad \sqrt{1-e^{-2 \phi}} \\
& \frac{d y}{d \phi} \quad-e^{-\phi}
\end{aligned}
$$

Le potentiel o la valeur $0=0$ dans le plan de l'ouverture d'écoulement et croît avec les abscisses $x$ à l'infini. D'après ce qui a été exposé antérieurement, il est évident que l'écoulement scrtant de l'ouverture au-dessous de la vanne dans le canal horizontal remplit également les 
conditions de l'écoulement avec potentiel comme l'écoulement par une ouverture dans le fond. Les mêmes équations 4 et 5 sont encore en vigueur pour la courbe d'écoulement conformément à la figure 1 avec les axes $x$ et $y$ tournés de $90^{\circ}$ par rapport à la figure 8 .

Conșidérons donc le jet sortant par l'ouverture au-dessous de la vanne comme la moitié symétrique d'un jet sortant d'une ouverture dans le fond. Tandis que dans le cas de l'écoulement par une ouverture dans le fond, nous choisissons la valeur de la fonction de courant $\psi_{1}=0$ et $\psi_{2}=\pi$ pour les deux lignes de courant extrêmes, prenons dans le cas de l'écoulement sortant audessous d'une vanne $\psi=0$ pour la ligne de courant extrême identique avec l'axe des $x$ et pour l'autre ligne de courant qui est justement la courbe d'écoulement $\psi_{2}=\frac{\tau_{0}}{2}$.

Dès lors, le débit entre les deux lignes de courant extrêmes par unité de longueur de fente

a comme valeur $\frac{\pi}{2}$

Pour l'unité de vitesse la distance des deux lignes de courant à l'infini est :

$$
y=\frac{\pi}{2}
$$

En intégrant les équations 4 et 5 , nous obtenons

$$
\begin{gathered}
x=k+\phi-\sqrt{1-e^{-2 \phi}}+\ln \left(1+\sqrt{1-e^{-2 \phi}}\right. \\
y=k_{2}+e^{-0}
\end{gathered}
$$

Pour $0=\mathrm{O}$ on a $\mathrm{x}_{0}=\mathrm{O}$; comme l'expression après la constante d'intégration $K_{1}$, dans l'équation (7) a, pour $\varphi=0$, la valeur 0 , on a aussi :

$$
K_{1}=0
$$

$$
\text { Pour } i=\infty \text { on a y } \infty=\frac{\pi}{2} \text {; comme le }
$$
deuxième membre de l'équation (8) a, pour $\hat{i}=\infty$, la valeur $\mathrm{O}$, on $a$ :

$$
K_{i}=\frac{\pi}{2}
$$

Afin de simplifier l'écriture, posons:

$$
\sqrt{1-e^{-2 \phi}}=A
$$

er exprimons les équations 7 et 8 à l'aide dés équations 9,10 et 11 sous la forme suivante:

$$
\begin{aligned}
& x=-A+\ln (1+A)(12) \\
& y=\frac{-}{2}+e^{-\%}
\end{aligned}
$$

Ensuite, pour $=0$, il résulte de l'équation $13:$

$$
y_{0}=\frac{\pi}{2}+1
$$

Par conséquent, la dimension a de l'ouverture d'écoulement est :

$$
a=y=\frac{\pi+2}{2}
$$

Nous appellerons le rapport:

$$
\frac{y}{a}=\alpha
$$

le coefficient de contraction.

Celui-ci a pour l'écoulement par l'ouverture au-dessous de la vanne la valeur théorique:

$$
\alpha=\frac{\frac{\pi}{2}}{\frac{\pi+2}{2}}=\frac{\pi}{\pi+2}
$$

$$
\text { Pour } \pi=\frac{22}{7} \text {, on } a:=\frac{11}{18}=0,61
$$

\section{Enoncé 4 : La valeur théorique du coefficient} de construction est constante, soit 0,61 .

Afin d'introduire la hauteur d'ouverture a de la vanne comme paramètre variable dans les équations 12 et 13 , multiplions ces dernières par l'équation 14 mise sous la forme suivante:

$$
\begin{aligned}
& \frac{1}{a}=\frac{2+\pi-\pi}{\pi+2}=1-\alpha=\beta=0 \\
& \text { Alors } \frac{x}{a}=\beta[\phi-A+\ln (1+A)]
\end{aligned}
$$

Désignons l'expression entre crochets de l'équation (18) par :

$$
f_{1}(\phi)=\phi-\sqrt{1-e^{-2 \phi}}+\ln \left(1+\sqrt{1-e^{-2 \cdot 5}}\right)
$$


Ensuite écrivons :

$$
\frac{x}{a}=x_{1}=\beta f_{1}(q)
$$

Dans les équations 18 et 20 , nous avons ordonné les variables de manière que les deux membres des équations soient un nombre sans dimensions $x_{1}$ qui indique le rapport entre l'abscisse $x$ et la hauteur d'ouverture a. Appelons le nombre $x$, le nombre caractéristique de l'abscisse $\mathbf{x}$, ou par abréviation, "le paramètre d'abscisse ». Si nous exprimons a en mètres, $x_{1}, m$. est la hauteur de l'abscisse de la courbe $T_{1}$ pour l'ouverture de la vanne $a_{1}$, c'est-à-dire $a_{1}=1 \mathrm{~m}$

Multiplions l'équation 13 par l'équation 17:

$$
\frac{y}{a}=\beta\left(\frac{\pi}{2}+e^{-i}\right)
$$

Nous gagnerons en clarté dans le tracé des courbes d'écoulement pour les différentes ouvertures de la vanne si nous choisissons comme origine $\mathrm{O}_{2}$ des axes de coordonnées la pointe du bord inférieur de la vanne (fig. 1) et si nous convenons que l'axe horizontal des $x$ est positif vers la droite et l'axe vertical des $t$ est positif vers le bas.

Pour les axes $x$ et $t$ les équations 18,19 et 20 ne varient pas. Comme a $-y=t$, l'équation 21 prend la forme

$$
\frac{a-y}{a}=\frac{t}{a}=1-\beta\left(\frac{\pi}{2}+e^{-i t}\right)
$$

Faisons ensuite une transformation à l'aide de l'équation (17)

$$
\frac{t}{a}=\beta\left(1-e^{-i}\right)
$$

Posons

et

$$
f_{2}(\varphi)=1-e^{-\%}
$$

$$
\frac{t}{a}=\beta f_{2}(\varphi)=t_{1}
$$

Appelons $t$, le "paramètre d'ordonnée " qui, en exprimant les longueurs en $\mathrm{m}$, pour $a_{1}=1 \mathrm{~m}$. donne l'ordonnée de la courbe $T_{1}$.

La mise sous forme de paramètre sans dimensions des variables dans les formules et les équations offre un grand avantage : les nombres sans dimensions sont valables pour tous les systèmes de mesure.

Les nombres $x_{1}, y_{1}$ ef $t$ sont des fonctions du potentiel o.

Pour les différentes valeurs du potentiel $\varphi$ et pour $a=1 \mathrm{~m}$., les nombres $x_{1}$ et $t_{1}$ sont indiqués dans le tábleau $A$.

Pour $=6$, nous trouvons dans ce tableau que pour l'ouverture $a=1 \mathrm{~m}$., la courbe $T_{1}$ baisse à la distance $x_{1}=2 \mathrm{~m} .2146 \mathrm{de}$ $t_{1}=0 \mathrm{~m} .388$, c'est-à-dire une valeur qui ne diffère pratiquement pas de la valeur $0 \mathrm{~m} .389$ à l'infini.

D'après les équations 18 et 22 , nous concluons :

Enoncé 5 : Les courbes théoriques $T$ de l'écoulement au-dessous de l'arête biseautée d'une vanne dans un canal horizontal dépendent de la hauteur a de l'ouverture; par contre, elles sont affines pour la même hauteur d'ouverture.

Enoncé 6 : Les points analogues des courbes $T$ pour différentes hauteurs a de l'ouverture sont déterminés par la même valeur du potentiel.

$11^{\circ}$ - Les tangentes de la courbe $T$.

Transcrivons l'équation 5 pour les axes $x$ et $t$ :

$$
\begin{aligned}
& t=a-y ; d t=-d y \\
& \frac{d t}{d_{\varphi}}=e^{-y}
\end{aligned}
$$

Le coefficient angulaire de la tangente $u$ à la courbe $T$ est d'après les équations 25 et 4 :

$$
\frac{d t}{d x}=\frac{1}{e^{\phi} \sqrt{1-e^{-2 \phi}}}=\frac{1}{e^{\phi} A}=\operatorname{tg} \theta
$$

L'angle $\theta$ est celui que forme la tangente avec l'axe des $x$.

Enoncé 7 : Les paints analogues des courbes $T$ ont des tangentes avec le même coefficient angulaire.

Pour $\varphi=0$, on a $A=0$; il résulte alors, de l'équation 26 :

$$
\operatorname{tg} \theta=\infty, 0=90^{\circ} .
$$

Enoncé 8 : La courbe $T$ a comme tangente au 
TABLEAU A

Les nombres théoriques de la courbe d'écoulement $T_{1}$, c'est-à-dire pour la hauteur d'ouverture $a=1 \mathrm{~m}$.

\begin{tabular}{|c|c|c|c|c|c|c|c|}
\hline 9 & 0 & 0,02 & 0,05 & 0,0622 & 0,07 & 0,10 & 0,12 \\
\hline$v$ & $90^{\circ}$ & & & $70^{\circ}$ & & & \\
\hline$x_{1}$ & 0. & 0,0011 & 0,0040 & 0,0056 & 0,0066 & 0,0112 & 0,0147 \\
\hline $\mathrm{t}_{1}$ & 0 & 0,0077 & 0,0190 & 0,0235 & 0,0263 & 0,0370 & 0,0440 \\
\hline$r_{1}$ & 0 & & & 0,1416 & & & \\
\hline$c_{1}$ & 0 & & & 0,1386 & & & \\
\hline$d_{1}$ & 0 & & & 0,0249 & & & \\
\hline
\end{tabular}

\begin{tabular}{|c|c|c|c|c|c|c|c|}
\hline$j$ & 0,15 & 0,20 & 0,2665 & 0,50 & 0,6931 & 1,00 & 1,30 \\
\hline$j$ & & & $50^{\circ}$ & & $30^{\circ}$ & & \\
\hline$x_{1}$ & 0,0204 & 0,0309 & 0,0467 & 0,1128 & 0,1754 & 0,2830 & 0,3936 \\
$t_{1}$ & 0,0542 & 0,0705 & 0,0910 & 0,1531 & 0,1945 & 0,2459 & 0,2830 \\
$r_{1}$ & & & 0,3264 & & 0,6737 & & \\
$c_{1}$ & & & 0,2967 & & 0,5123 & & \\
$d_{1}$ & & & 0,1188 & & $-0,3890$ & & \\
\hline
\end{tabular}

\begin{tabular}{|c|c|c|c|c|c|c|c|c|c|}
\hline 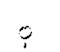 & 1,7507 & 2,00 & 2,50 & 3,00 & 3,50 & 4,00 & 5.00 & 6,00 & \\
\hline v & $10^{\circ}$ & & & & & & & & $0^{\circ}$ \\
\hline$x_{1}$ & 0,5646 & 0,6604 & 0,8597 & 1,0479 & 1,2305 & 1,4367 & 1,8256 & 2,2146 & 0,3890 \\
\hline$t_{1}$ & 0,3214 & 0,3364 & 0,3571 & 0,3696 & 0,3773 & 0,3819 & 0,3864 & 0,3880 & \\
\hline$r_{1}$ & 2,2060 & & & & & & & & \\
\hline$c_{1}$ & 0,9477 & & & & & & & & \\
\hline$d_{1}$ & $-1,8511$ & & & & & & & & \\
\hline
\end{tabular}


départ de l'origine l'axe des t. Pour $\varphi=\infty$, on $a$, d'après l'équation 26 :

$$
\operatorname{tg} 0=0 \quad \theta=0
$$

\section{Enoncé $9:$ La courbe $T$ tend vers une asymp-} tote horizontale à l'intini.

Nous obtiendrons l'équation de cette asymptote à l'aide de l'équation 22 pour $0=\infty$.

$$
\mathrm{t} \infty=\mathrm{a}
$$

Nous verrons par la suite que les courbes théoriques $T$ peuvent être utilisées pour la résolution de notre problème. Pour cette raison, il est utile que nous connaissions tout angle déterminé $\theta$ de la tangente $u$, la valeur du potentiel 0 et la coordonnée du point correspondant $U$.

Nous tirerons facilement, de l'équation 26:

$$
\phi=\frac{\log \left(\frac{1}{\operatorname{tg} 2 \theta}+1\right)}{2 \log e}
$$

On trouvera, dans le tableau $A$, les valeurs du potentiel is et les paramètres de coordonnées $x_{1}$ et $t_{1}$ du point correspondant pour l'angle $s$ de la tangente avec l'axe des $x$ ayant les valeurs $90^{\circ}$, $70^{\circ}, 50^{\circ}, 30^{\circ}, 10^{\circ}$ et $0^{\circ}$.

\section{$12^{\circ}$ Détermination graphique des courbes $T$ pour différentes ouvertures a de la vanne.}

Traçons pour la figure 10 la courbe $T_{1}$ pour $a_{1}=1 \mathrm{~m}$ d'après les nombres $x_{1}$ et $t_{1}$ du tableau A. Prenons sur cette courbe le point $U_{1}$ $\left(x_{1}, t_{1}\right)$. Au point $U_{1}$ correspond le point $U_{0},{ }_{6}$ de la courbe $T_{0,6}$ pour a $=0,6 \mathrm{~m}$.

Traçons sur la fig. 10, par le foint marqué 0,6, une parallèle à $1.0 . U_{1}$. Relions par une droite $U_{1}$ avec $\mathrm{O}_{2}$. Le point d'intersection $\mathrm{U}_{0 \cdot 6}$ est le point recherché, car.

$$
a_{0 \cdot 6}: a_{1}=t_{0 \cdot 6}: t_{1}=x_{0 \cdot 6}: x_{1}
$$

Les tangentes des deux points analogues sont parallèles :

$$
u_{0.6} / / u_{1}
$$

ainsi, d'après une courbe $T$ tracée, nous déduisons rapidement d'autres courbes pour différentés ouvertures par interpolation ou extrapolation.

\section{$13^{\circ}$ Les rayons de courbure de la courbe $T$.}

Conformément à l'énoncé 8 , la courbe $T$ a comme tangente au départ à l'origine l'axe des $t$. Le courbe réelle d'écoulement n'a pas la même allure; la ligne de courant correspondante sort du plan de la vanne pour contourner l'arête biseautée de la vanne moyennant un arc avec

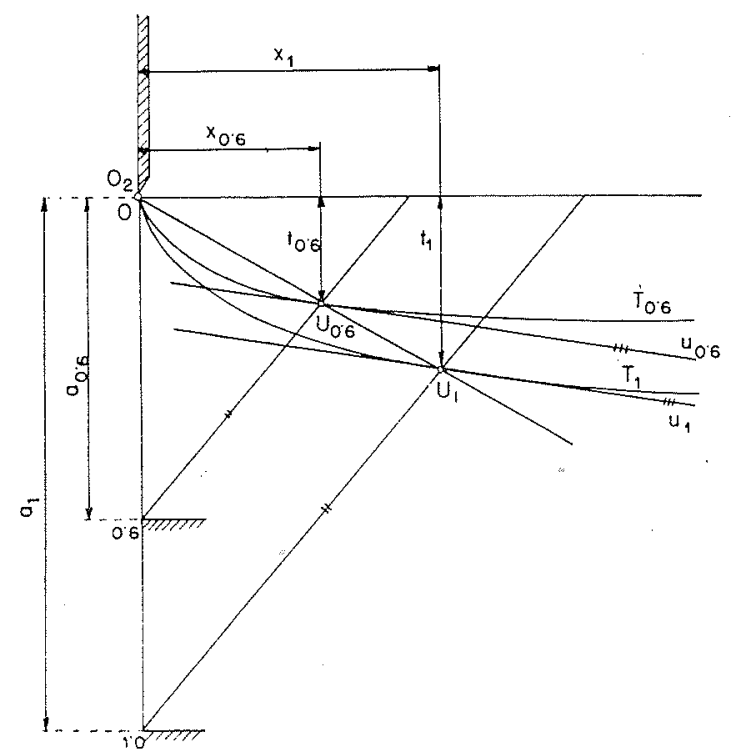

fig. 10 Détermination des courbes d'écoulement aux différentes ouvertures

une courbure de grandeur finie. Examinons pour cette raison encore la courbe $T$ en ce qui concerne la courbure.

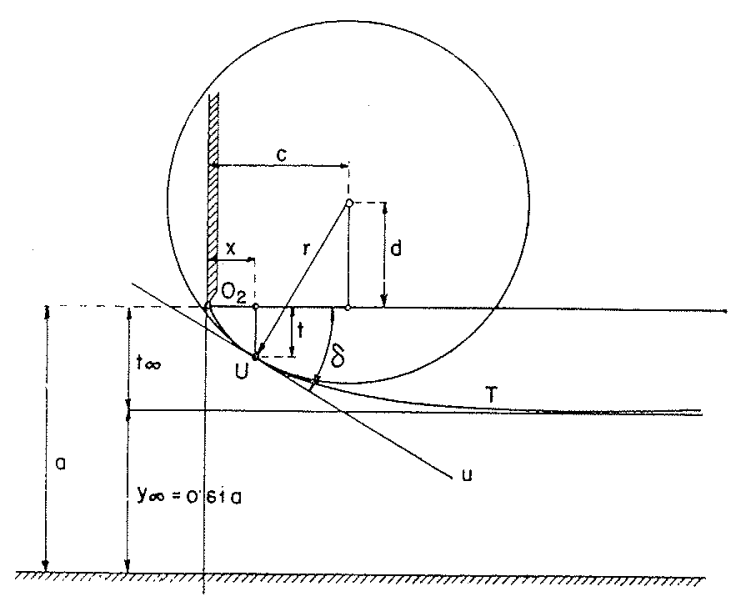

fig. 11

La tangente ef le rayon de courbure d'une courbe d'écoulement

Pour la courbe donnée par les équations de forme 20 et 24 , que nous transcrivons sous la forme suivante:

$$
\begin{aligned}
& x=a \beta f_{1}(\varphi) \\
& t=a \beta f_{2}(\varphi)
\end{aligned}
$$

calculons le rayon de courbure $r(10)$ d'après l'expression :

$$
r=\frac{\left\{a^{2} \beta^{2}\left[\left(f_{1}^{\prime}\right)^{2}+\left(f_{2}^{\prime}\right)^{2}\right]\right\}^{3 / 2}}{a^{2} \beta^{2}\left(f_{1}^{\prime} f_{2}^{\prime \prime}-f_{1}^{\prime \prime} f_{2}^{\prime}\right)}
$$


ou sous forme de nombres sans dimensions:

$$
\frac{r}{a}=\beta \frac{\left[\left(f_{1}^{\prime}\right)^{2}+\left(f_{2}^{\prime}\right)^{2}\right]^{3 / 2}}{f_{1}^{\prime} f_{2}^{\prime \prime}-f_{1}^{\prime \prime} f_{2}^{\prime}}
$$

$f_{1}^{\prime}, f_{2}^{\prime}, f^{\prime \prime}{ }_{1}, f^{\prime \prime}{ }_{2}$ sont les dérivées premières et secondes des fonctions $f_{1}(0)$ et $f_{2}(\varphi)$ par rapport à la variable qui est le potentiel.

Si nous tenons compte de l'équation 19, nous avons l'expression:

$$
f_{1}^{\prime}=\frac{d f_{1}}{d \phi}=1-\frac{e^{-2 \phi}}{\sqrt{1-e^{-2 \phi}}}+\frac{e^{-2 \phi}}{\sqrt{1-e^{-2 \phi}\left(1+\sqrt{1-e^{-2} \phi^{\prime}}\right.}}
$$

qui, après une simple transformation, donne:

$$
\begin{aligned}
& f_{1}^{\prime}=\sqrt{1-e^{-2 \phi}}=A \quad \ldots 35 \\
& f=\frac{d f_{1}^{\prime}}{d \phi}=\frac{e^{-2 \phi}}{\sqrt{1-e^{-2 \phi}}}=\frac{1}{e^{-2 \phi} A} \ldots 36
\end{aligned}
$$

Si nous partons de l'équation 23 , nous trouvons:

$$
\begin{aligned}
& f_{2}^{\prime}=\frac{d f_{2}}{d \phi}=e^{-2 \phi} \quad \ldots 37 \\
& f_{2}^{\prime \prime}=\frac{d f_{2}^{\prime}}{d \phi}=-e^{-\phi} \quad \ldots 38
\end{aligned}
$$

En introduisant les équations 35 à 38 dans l'équation 34 , on a :

$$
\frac{1}{a}=\beta \frac{\left(1-e^{-2 \phi}+e^{-2 \phi}\right)^{3 / 2}}{-e^{-\phi} \sqrt{1-e^{-2} \phi^{-}}-e^{-\phi}} \frac{e^{-2 \phi}}{\sqrt{1-e^{-2} \phi}}
$$

Après une petite transformation, on obtient :

$$
\left.\begin{array}{rl}
\frac{r}{a} & =-\beta e^{\phi} \sqrt{1-e^{-2 \phi}} \\
& =-\beta e^{\phi} A=-r_{1}
\end{array}\right\} \ldots 39
$$$$
\text { ou } r_{1}=\beta e^{\phi} A
$$

Les nombres sans dimensions $r_{1}$ du rayon de courbure où les longueurs en $m$ du rayon de courbure $r$ pour l'élévation de la vanne $a=1 \mathrm{~m}$ sont indiqués dans le tableau $A$ et cela pour les points de contact des tangentes de pente qui $y$ est également indiquée. Pour d'autres valeurs a les rayons de courbure correspondants sont calculés en multipliant les nombres $r_{1}$ par la valeur a donnée en $\mathrm{m}$.
Comme pour $0=0$ on $a: A=0$, il résulte de l'équation 39: $r_{0}=0$

Pour $0=\infty$, on a e $0=\infty$ et d'après la même équation:

$$
r \infty=-\infty
$$

Enoncé 10: Le rayon de courbure $r$ de la courbe $T$ croît avec la valeur du potentiel et avec la grandeur de l'élévation a de la vanne. Les rayons de courbure aux points analogues pour différentes ouvertures a de la vanne sont, par contre, directement proportionnels à la valeur a.

Enoncé 11: La courbe d'écoulement $T$ a sur l'arête de l'ouverture d'écoulement un rayon de courbure infiniment petit. Toutefois le rayon de courbure de la courbe d'écoulement réelle doit avoir à l'endroit cité une grandeur finie.

\section{$14^{\circ}$ Coordonnées du centre de la courbure de la courbe $T$.}

L'abscisse c et l'ordonnée d (fig. 11) du centre de la courbure des courbes suivant les équations 32 et 33 sont données par les expressions suivantes, si on leur donne la forme de nombres sans dimensions:

$$
\begin{aligned}
& \frac{c}{a}=\beta\left[f_{1}-f_{2}^{\prime} \frac{\left(f_{1}^{\prime}\right)^{2}+\left(f_{2}^{\prime}\right)^{2}}{f_{1}^{\prime} f_{2}^{\prime \prime}-f_{1}^{\prime \prime} f_{2}^{\prime}}\right] \\
& \frac{d}{a}=\beta\left[f_{2} f_{1}^{\prime} \frac{\left(f_{1}^{\prime}\right)^{2}+\left(f_{2}^{\prime}\right)^{2}}{f_{1}^{\prime} f_{2}^{\prime \prime}-f_{1}^{\prime \prime} f_{2}^{\prime}}\right]
\end{aligned}
$$

Introduisons les valeurs des dérivées premières et secondes des fonctions $f_{1}$ et $f_{2}$ des équations 35 à 38 dans la fraction du deuxième membre entre parenthèses carrées de l'équation 43

$$
\frac{1-e^{-2 \phi}+e^{-2 \phi}}{-e^{-\phi} \sqrt{1-e^{-2 \phi}}-e^{-\phi} \frac{e^{-2 \phi}}{\sqrt{1-e^{-2 \phi}}}}
$$

$$
=-e^{\phi} \sqrt{1-e^{-2 \phi}}
$$

Introduisons l'expression (a) dans l'équation 43 , où en même temps nous introduisons, d'après l'équation 20: $B f_{1}=x_{1}$.

$$
\begin{aligned}
\frac{c}{a} & =x_{1}+\beta e^{-\phi} e^{\phi} \sqrt{1-e^{-2 \phi}} \ldots 45 \\
& =x_{1}+\beta A=c_{1}
\end{aligned}
$$


De même, introduisons dans l'équation 44 l'expression (a) et d'après l'équation 24

$$
B f_{2}=t_{1} \text {. }
$$

$$
\begin{aligned}
\frac{a}{d} & =t_{1}-\beta \sqrt{1-e^{-2 \phi}} e^{\phi} \sqrt{1-e^{-2 \phi}} \\
& =t_{1}-\beta e^{\phi}\left(1-e^{-2 \phi}\right) \\
\therefore & =t_{1}-\beta e^{\phi} A^{2}=d_{1}
\end{aligned}
$$

Pour $0=\mathrm{O}$ on a $\mathrm{A}=\mathrm{O}, \mathrm{x}_{1}=\mathrm{O}, \mathrm{t}_{1}=\mathrm{O}$; alors on 'a aussi $c_{1}=0, d_{1}=0$; de même, il se confirme que $r_{0}=0$

Les nombres $c_{1}$ et $d_{1}$ sont donnés dans le tableau $A$.

\section{$15^{\circ}$ L'équation de la courbe d'écoulement pour un liquide visqueux.}

La courbe d'écoulement réelle S s'établit par rapport à la courbe théorique $\mathrm{T}$ de manière que son point le plus bas a les coordonnées $x_{n}$ et $s^{n}$ (fig. 1) de grandeurs finies.

Admettons que la courbe théorique $\mathrm{T}$ calculée en se basant sur la théorie des fonctions analytiques, exprime la loi exacte du mouvement d'un liquide non visqueux dans une conduite idéale; alors la courbe d'écoulement réelle $S$ pourra être déduite de la courbe $T$ si on introduit dans les équations de la courbe $T$ l'expression de la résistance qui s'oppose au mouvement d'un liquide visqueux dans une conduite rugueuse.

Exprimons cette résistance par une chute relative 1. Tandis que la courbe théorique $T$ baisse pour l'abscisse $x$ à l'ordonnée $t$, la courbe réelle $S$ baisse pour la même abscisse $x$ à l'ordonnée s. Etant donné que la vitesse réelle du courant d'eau doit être inférieure à la vitesse correspondante d'un liquide non visqueux, on doit avoir $s<t$.

Nous pouvons alors exprimer l'ordonnée $s$ comme suit :

$$
\mathrm{s}=\mathrm{t}-\mathrm{Jx} \quad \ldots 48
$$

ou pour avoir toujours affaire à des nombres sans dimensions pour la raison signalée déjà au paragraphe 10 :

$$
\frac{s}{a}=t_{1}-J x_{1}=s_{1} \ldots 449
$$

Pour les abscisses $x$ de la courbe $S$ l'équation 18 est vraie comme pour les abscisses de la courbe $T$.
Les équations 48 et 49 disent:

Enoncé 12 : Si la courbe théorique $T$ traduit effectivement la loi de mouvement pour les conditions idéales, les courbes d'écoulement réelles $S$ se trouvent du côté intérieur des courbes $T$.

Supposons qu'on puisse exprimer en quelque manière la chute J sur la longueur de l'abscisse $x$ par la valeur constante J. Alors il est possible de déterminer les coordonnées $x$ et $s$ comme coordonnées du point le plus bas de la courbe $S$ qui a une tangente horizontale.

Pour les coefficients angulaires de la tangente horizontale de la courbe $S$ on a:

$$
\frac{d s}{d x}=\frac{\frac{d s}{d \phi}}{\frac{d x}{d \phi}}=0
$$

II résulte de l'équation 48 :

$$
\frac{d s}{d \phi}=t^{\prime}-J x^{\prime}
$$

Etant donné que :

$$
\begin{aligned}
& t^{\prime}=a \xi f_{2}^{\prime}(p) \\
& x^{\prime}=a \beta f_{i}^{\prime}(p)
\end{aligned}
$$

on a, en utilisant les équations 37 et 35 :

$$
\begin{array}{ll}
\frac{d s}{d \phi}=a \beta\left(e^{-\phi}-J_{s} A\right) & \ldots 50 \\
\frac{d x}{d \phi}=a \beta A & \ldots 51 \\
\frac{d s}{d x}=\frac{e^{-\phi}-J_{s} A}{A}=\frac{1}{e A}-J_{s} \ldots 52 \\
\text { Pour } \frac{d s}{d x}=0,
\end{array}
$$

on a:

$$
\frac{1}{e^{\phi} \sqrt{1-e^{-2 \phi}}}=J_{s} \ldots 53
$$

d'où la valeur du potentiel un pour le point le plus bas de la courbe $S$

$$
\phi_{n}=\frac{\log \frac{1}{J_{s}^{2}}+1 \ldots 54}{2 \log e}
$$

Si nous comparons les formules 54 et 30 , nous pouvons formuler cet énoncé : 
Enoncé 13: Le point le plus bas de la courbe d'écoulement réelle $S$ a l'abscisse $x_{n}$ en commun avec le même point de la courbe $T$, et sa tangente a le coefficient angulaire tg $\rho$ de même grandeur que la valeur $J_{s}$ de la chute, suivant l'abscisse $x$.

Par conséquent, l'équation 18 pour l'abscisse $x=$ du point le plus bas de la courbe $S$ est :

$$
\begin{aligned}
& \frac{x_{n}}{o}=\beta\left[\phi_{n}-\sqrt{1-e^{-2 \phi_{n}}}+1_{n}\left(1+\sqrt{1-e^{-2 \phi_{n}}}\right)\right] \\
& \text {... } 55
\end{aligned}
$$

Nous calculerons l'ordonnée au moyen des équations 49,22 et 55 :

$$
\frac{s_{n}}{a}=\beta\left(\cdot \delta^{-\phi_{n}}\right)-j_{s} \frac{x_{n}}{a} \ldots 56
$$

Le coefficient de contraction $\alpha_{s}$ pour l'écoulement d'un liquide visqueux est donc donné par l'expression:

$$
a_{n}=\frac{a-s_{n}}{a}=1-\frac{s_{n}}{a} \ldots 57
$$

dans laquelles est tiré des équations 56 et 55 .

La valeur théorique du coefficient de contraction est :

$$
a=1-\frac{+\infty}{a}
$$

Comme on a évidemment : $s_{n}<t \alpha$ on a manifestement aussi $\%$ s $>\alpha 9$

Enoncé 14: Le coefficient de contraction $\alpha_{\mathrm{s}}$ d'un liquide visqueux a une valeur plus grande que le coefficient de contraction d'un liquide non visqueux.

L'énoncé 14 découle aussi directement de l'énoncé 12.

\section{$16^{\circ}$ Les courbes d'écoulement et les coefficients de contraction aux différentes charges relatives $h_{1}$.}

Tout ce qui a été déduit théoriquement jusqu'ici est valable pour des ouvertures d'écoulement petites par rapport à la retenue en amont de la vanne.

Mais si la charge relative $h_{1}$ a une faible valeur, les courbes de contraction et de ce fait les coefficients de contraction sont différents. Plus la valeur $h_{1}$ est faible, plus la vitesse d'arrivée à la vanne joue un grand rôle et d'autant plus varie la figure des lignes de courant d'approche.
A. $\mathrm{KOCH}(14)$ a essayé de donner une théorie des coefficients de contractions pour les grandes et les petites valeurs de $h_{1}$, et ceci en prenant en considération toutes les forces qui agissent lors du passage de l'eau de dessous la vanne dans un canal horizontal. C'est aussi en tenant compte de ces forces que $\mathrm{KOCH}$ avait déterminé les ordonnées de la courbe d'écoulement comme l'ordonnée du point de tangence pour différentes inclinaisons de la tangente à cette courbe. Mais il n'a pas réussi à déterminer les abscisses correspondantes de la courbe d'écoulement. Dans son ouvrage, $\mathrm{KOCH}$ donne plusieurs valeurs du coefficient de contraction pour des faibles valeurs du rapport $h_{1}$ calculées d'après sa formule.

Mais les valeurs données par $\mathrm{KOCH}$ diffèrent beaucoup des valeurs que j'ai trouvées expérimentalement, comme on le verra au paragraphe 20. Pour cette raison, nous ne parlerons pas des considérations théoriques de $\mathrm{KOCH}$.

$17^{\circ}$ Les valeurs théoriques du coefficient de contraction pour l'écoulement au-dessous de la surface d'appui de la vanne ayant la forme de la courbe d'écoulement théorique.

Si nous donnons à la surface d'appui de la vanne la forme d'une partie de la courbe $T$, il s'établit un écoulement par l'ouverture de hauteur a-t qui, théoriquement, se rétrécit à la hauteur 0,61 a.

Le coefficient de contraction est done donné par le rapport

$$
a_{p}=\frac{0,61 a}{a-t}=0,61 \frac{1}{1-t_{1}} \quad \ldots .
$$

ou $t_{1}$ est donné par l'équation 24 et le tableau numérique $A$.

Par exemple, pour la surface terminée par une tangente ayant une inclinaison de $10^{\circ}$, on a, d'après le tableau $A, t_{1}=0,3214$, et alors

$$
\alpha \mathrm{p}=0,90
$$

Pour une surface exécutée jusqu'à la valeur ciu potentiel $\rho=6$, on $a \propto p=1$.

Enoncé 15 : La valeur théorique $\alpha p$ du coefficient de contraction de l'écoulement audessous d'une surface formée suivant la courbe $T$, crôit avec la valeur du porentiel o valable pour l'extrémité de la surface depuis la valeur 0,6 l jusqu'à la valeur 1 .

(à suivre). 